\title{
Brazilian Journal

\section{CELLULOSE ACETATE-BASED MOLECULARLY IMPRINTED POLYMERIC MEMBRANE FOR SEPARATION OF VANILLIN AND O-VANILLIN}

\author{
Chunjing Zhang, Shian Zhong ${ }^{*}$ and Zhengpeng Yang \\ College of Chemistry and Chemical Engineering, Phone: 0731-8830416, \\ Fax: 0731-8879616, Central South University, Changsha, 410083, China. \\ E-mail: zhongshian@yahoo.com.cn \\ (Received: October 28, 2006; Accepted: July 13, 2007)
}

\begin{abstract}
Cellulose acetate-based molecularly imprinted polymeric membranes were prepared using vanillin as template molecule. The microscopic structure of the resultant polymeric membranes was characterized by SEM and FTIR spectroscopy, and the selective binding properties and separation capacity of the membranes for vanillin and o-vanillin were tested with binding experiments and separate experiments, respectively. The results showed that the vanillin-imprinted polymeric membranes displayed higher binding and separation capacity for vanillin in comparison with the non-imprinted polymeric membranes; the maximum binding amount $\mathrm{Q}_{\max }$ for vanillin is $387.3 \mu \mathrm{mol} / \mathrm{g}$ and the selectivity factor is 3.82 for vanillin/o-vanillin. In addition, the effect of cross-linker concentration on the binding of vanillin on the imprinted polymeric membranes was also evaluated and the optimal concentration is $320 \mathrm{mM}$.

Keywords: Cellulose acetate (CA); Molecularly imprinted polymeric membrane; Vanillin; O-vanillin; Separation.
\end{abstract}

\section{INTRODUCTION}

Over the past years, molecularly imprinting polymers (MIPs) have been investigated widely (Xu et al., 2004), and have shown unique advantages over natural biological receptors in terms of physical and chemical stability and the possibility of using MIPs in harsh environmental conditions (Hilal and Kochkodan, 2003). Usually, MIPs are produced in non-aqueous solutions by bulk polymerization of functional and cross-linker monomers in the presence of template molecules. After removal of the template molecules, the MIP retains receptor sites that are complementary to the template molecule in shape and position of the functional groups. In this way, molecular memory is produced in the polymer, making the MIPs capable of selectively rebinding the template molecule (Vlatakis et al., 1993, Cormack and Mosbach, 1999). Based on imprinting effect of the MIPs, MIPs have been employed in many fields, such as chromatographic separation (Sellergren et al., 1998, Brauner and Shacham, 1999, Fan et al., 2003), recognition of elements in biological sensors (Kroger et al., 1999, Jakusch et al., 1999), solid phase extraction (Mullett and Lai, 1998, Kugimiya and Takeuchi, 1999, Jodlbauer et al., 2002), receptor mimics in biological systems (Zhou et al., 1998) and so on. However, the practical application of MIPs is still under development (Haupt and Mosbach, 2000). Recently, MIP membranes prepared by photoinitiated surface modification of microfiltration membranes in the presence of template molecules have attracted much research interest due to the ability to change their diffusive permeability automatically by responding to the template molecules (Piletsky et al., 2000, Kochkodan et al., 2001). The MIP membranes have shown selective adsorption of target molecules (Takeda and Kobayashi, 2006), and the technique has also been applied to other research fields, which focus on

*To whom correspondence should be addressed 
separation of food (Trotta et al., 2002) and medicine (Yoshikawa et al., 2006).

In the present work, the imprinting technique has been extended to prepare selective MIP membranes on the surface of cellulose acetate (CA) membranes using vanillin as template molecules (namely CAMIP membranes). The microscopic structure of the imprinted membranes was characterized, and the binding properties and permeation selectivity of the membranes for separation of vanillin and o-vanillin were investigated.

\section{EXPERIMENTAL}

\section{Materials and Instruments}

Cellulose acetate (CA) membranes were purchased from Aldrich Chemical Co. Vanillin and o-vanillin were obtained from Sigma Chemical Co. Methacrylic acid (MAA), 2,2-Azobisisobutyronitrile (AIMN) and ethylene glycol dimethacrylate (EGDMA) were purchased from Aldrich. EGDMA and MAA were distilled to remove the inhibitor prior to use. Acetonitrile and methanol were of chromatographic grade. All other chemicals were of analytical grade and used without further purification.

UV spectrophotometer (DMS 80/90, Varian Techtron, Australia), Protégé 460 FTIR spectrometer (Nicolet Instrumental Corp.), JSM-5310LVB scanning electron microscopy (SEM) (JOEL, Japan) were employed for the spectroscopic measurements.

\section{Preparation of the CA-MIP Membranes}

The vanillin-imprinted CA-MIP membranes were prepared according to the following procedure. 7.61 $\mathrm{mg}(0.05 \mathrm{mmol})$ of vanillin was dissolved in $5 \mathrm{ml}$ acetonitrile, then $34.40 \mathrm{mg}(0.4 \mathrm{mmol}) \mathrm{MAA}, 0.33$ $\mathrm{ml}(1.7 \mathrm{mmol})$ EGDMA and $5 \mathrm{mg}$ AIBN were added to the vanillin solution, respectively. The mixture was degassed with an ultrasonic oscillator in an icewater bath for $10 \mathrm{~min}$. The $\mathrm{CA}$ membrane was placed into the above solution and stored in an icebox for $20 \mathrm{~min}$. The CA membrane was then taken from the mixture, placed between two glass plates and compressed in order to remove the air bubbles. A $300 \mathrm{~W}$ UV lamp was employed to illuminate the membrane for $6 \mathrm{~h}$ at about $4^{\circ} \mathrm{C}$. After the glass plates were separated, the CA-MIP membrane was washed repeatedly with methanol/acetic acid $(9: 1 \mathrm{v} / \mathrm{v})$ solution until the template molecule vanillin could not be detected by
UV-vis spectroscopy and then washed with methanol and kept in methanol at $4^{\circ} \mathrm{C}$.

The CA-NMIP membranes were prepared in the same way, but without the addition of the imprint molecule vanillin and worked up by the same procedure.

The morphology of the surface of the polymer membranes supported on the CA membrane was observed by scanning electron microcopy (SEM). The structural changes of the CA-MIP membrane were evaluated from FT-IR spectra in comparison with the structures of the blank CA membrane and vanillin-imprinted MIP powder.

\section{Binding Experiments}

The vanillin-imprinted CA-MIP membranes were immersed in $2.0 \mathrm{~mL}$ acetonitrile solution with template molecule vanillin at $25{ }^{\circ} \mathrm{C}$ for $24 \mathrm{~h}$ under constant stirring. The concentration of vanillin was measured by UV spectrophotometry. The amount Q $(\mu \mathrm{mol})$ of vanillin bound to the imprinted membranes can be calculated according to the concentration change of vanillin in solution before and after binding experiments. Q is the average value of three experiments.

\section{Evaluation of Membrane Separation Properties}

For separation tests, the polymeric membranes were mounted tightly between the two stirred chambers in an H-shaped two-compartment cell with constant stirring and permeation properties were examined at $25^{\circ} \mathrm{C}$. A $50 \mathrm{~mL}$ methanol solution containing $1.00 \mathrm{mM}$ vanillin and $1.00 \mathrm{mM}$ o-vanillin was placed in the feeding chamber, and $50 \mathrm{~mL}$ pure methanol in the receiving chamber. At an interval of 2 hours, the amount of each diffused analyte was determined by UV spectrophotometry.

\section{RESULTS AND DISCUSSION}

\section{Characteristics of Membranes}

When a blank CA membrane was soaked in the prepolymerization solution, some self-assembly complexes were loaded into the pores of the CA membrane. After polymerization and removal of the template molecules, an imprinted polymeric membrane attached to the CA membrane was achieved. It was observed that the modified membrane was smooth and flexible, and no obvious 
difference in macrography between CA membrane and CA-MIP membrane. However, obvious differences between the membranes were detected by scanning electron microscopy (Fig. 1). As seen in Fig. 1, the CA membrane possessed regular fibrous structure (Fig. 1a), while significant change was observed in its morphology after it interacted with the prepolymerization solution. The fibrous structure of the CA membrane disappeared and polymeric membrane was formed at its surface (Fig.1b), showing that the reaction between $\mathrm{CA}$ membrane and prepolymerization solution was really reliable. According to the surfaces and cross-sections of membranes observed by SEM, it was estimated that the thickness of the CA-MIP membrane was $35 \mu \mathrm{m}$, which was a little higher than that of the CA-NMIP membrane. This may be attributed to the presence of vanillin in the process of reaction, facilitating the formation of complexes at the surface of CA. In addition, the pore density of the CA-MIP membrane was higher due to the removal of the template molecule, suggesting a high porosity.
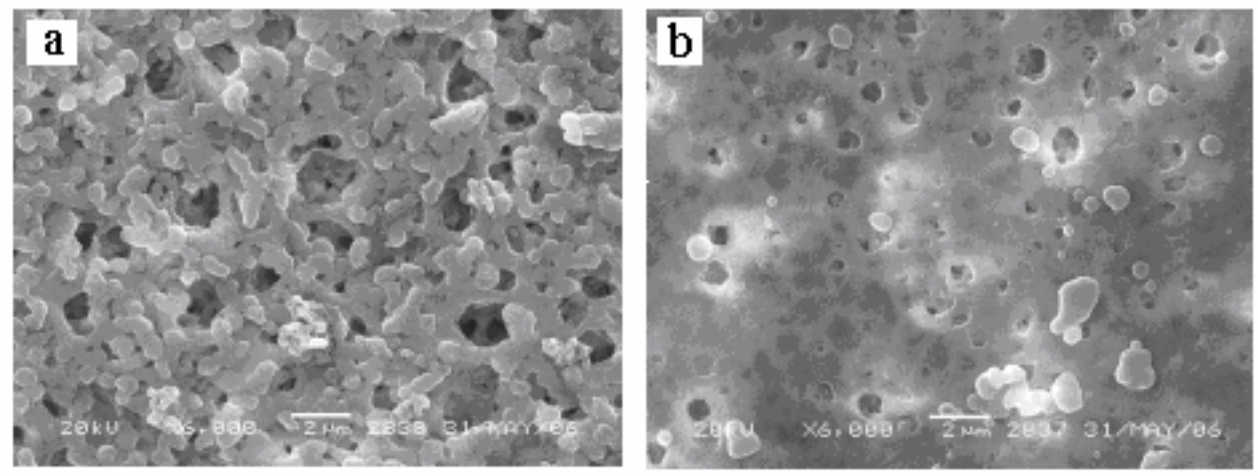

Figure 1: SEM images of (a) blank CA membrane and (b) CA-MIP membrane.

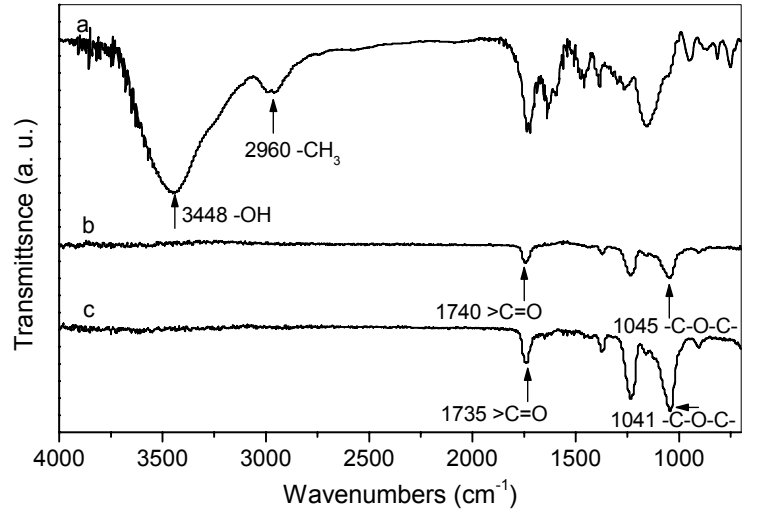

Figure 2: IR spectra of (a) MIP powder, (b) CA membrane and (c) CA-MIP membrane.

In order to confirm the interaction between MIP and CA membrane, IR spectra of (a) MIP powder, (b) CA membrane and (c) CA-MIP membrane were measured (Fig. 2). In the spectrum of the CA membrane, the IR bands at 1740 and $1045 \mathrm{~cm}^{-1}$ were attributed to the $>\mathrm{C}=\mathrm{O}$ stretch and $-\mathrm{C}-\mathrm{O}-\mathrm{C}$ - stretch, respectively. In the spectrum of the $\mathrm{CA}-\mathrm{MIP}$ membrane, it was found that the $>\mathrm{C}=\mathrm{O}$ and $-\mathrm{C}-\mathrm{O}-\mathrm{C}-$ stretching bands were shifted to shorter wavenumber; the $>\mathrm{C}=\mathrm{O}$ stretching band changed from $1740 \mathrm{~cm}^{-1}$ to $1735 \mathrm{~cm}^{-1}$ and the $-\mathrm{C}-\mathrm{O}-\mathrm{C}$ - stretching band changed from $1045 \mathrm{~cm}^{-1}$ to $1041 \mathrm{~cm}^{-1}$. In addition, it was also noted that the $-\mathrm{OH}$ stretching band at $3448 \mathrm{~cm}^{-1}$ and the $-\mathrm{CH}_{3}$ stretching band at $2960 \mathrm{~cm}^{-1}$ for MIP powder disappeared in the CA-MIP membrane. These changes in the IR spectra suggested that there is interaction between MIP and CA membrane.

\section{Study on the Interaction Between Template Molecule (Vanillin) and Functional Monomer (MAA)}

Because UV-vis spectrophotometry has been successfully used to investigate the interaction between the template molecule and the functional monomer (Chen et al., 2006), we employed it to 
study the interaction between vanillin and MAA. Assuming that the concentration of vanillin is $\mathrm{a}_{0}$, and the concentration of MAA is $b_{0}$ and that the analytical concentration of MAA is greater than that of vanillin, the binding reaction can be expressed as:

$\mathrm{V}+\mathrm{nM} \rightleftarrows \mathrm{W}$

where $\mathrm{V}$ and $\mathrm{M}$ represent the reactants vanillin and MAA respectively; $W$ represents the complex formed between them and $\mathrm{n}$ is the stoichiometric coefficient of $\mathrm{M}$. At equilibrium, the association constant can be written as

$$
\mathrm{K}=[\mathrm{W}] /[\mathrm{V}][\mathrm{M}]^{\mathrm{n}}
$$

According to mass balances, one has that:

$[\mathrm{V}]+[\mathrm{W}]=\mathrm{a}_{0}$

$[\mathrm{M}]+\mathrm{n}[\mathrm{W}]=\mathrm{b}_{0}$

Because $b_{0}>>a_{0}$, then [W] in Eq. (4) can be ignored. Thus,

$[\mathrm{M}] \approx \mathrm{b}_{0}$

By substituting Eqs. (3) and (5) into Eq. (2), we obtain:

$$
[\mathrm{W}]=\mathrm{a}_{0} \mathrm{~b}_{0}{ }^{\mathrm{n}} \mathrm{K} /\left(1+\mathrm{b}_{0}{ }^{\mathrm{n}} \mathrm{K}\right)
$$

According to the Lambert-Beer law, the absorbance of the solution is

$$
\begin{aligned}
& \mathrm{A}=\varepsilon_{\mathrm{V}} \mathrm{l}[\mathrm{V}]+\varepsilon_{\mathrm{M}} 1[\mathrm{M}]+\varepsilon_{\mathrm{W}} \mathrm{l}[\mathrm{W}] \\
& =\varepsilon_{\mathrm{V}} \mathrm{la}_{0}+\varepsilon_{\mathrm{M}} \mathrm{lb} \mathrm{b}_{0}+\left(\varepsilon_{\mathrm{W}}-\varepsilon_{\mathrm{V}}-\mathrm{n} \varepsilon_{\mathrm{M}}\right) 1[\mathrm{~W}]
\end{aligned}
$$

Defining $\mathrm{A}_{0, \mathrm{~V}}=\varepsilon_{\mathrm{V}} \mathrm{la}_{0}, \mathrm{~A}_{0, \mathrm{M}}=\varepsilon_{\mathrm{M}} \mathrm{l}_{0}$, then:

$$
\begin{aligned}
& \Delta \mathrm{A}=\mathrm{A}-\mathrm{A}_{0, \mathrm{~V}}-\mathrm{A}_{0, \mathrm{M}}=\left(\varepsilon_{\mathrm{W}}-\varepsilon_{\mathrm{V}}-\mathrm{n} \varepsilon_{\mathrm{M}}\right) 1[\mathrm{~W}] \\
& =\Delta \varepsilon \mathrm{l}[\mathrm{W}]
\end{aligned}
$$

Combination of Eq. (6) and Eq. (8) leads to Eq. (9):

$\Delta \mathrm{A} / \mathrm{b}_{0}{ }^{\mathrm{n}}=-\mathrm{K} \Delta \mathrm{A}+\mathrm{K} \Delta \varepsilon \mathrm{la}_{0}$

Where $A_{0, \mathrm{~V}}$ and $A_{0, \mathrm{M}}$ can be calculated from the spectra of pure acetonitrile solutions of vanillin and MAA, respectively. $\mathrm{n}$ is a simple positive integer. For $\mathrm{n}=1,2,3$, the plots of $\Delta \mathrm{A} / \mathrm{b}_{0}{ }^{\mathrm{n}}$ versus $\Delta \mathrm{A}$ are presented in Fig. 3. Because the wavelength employed in UV-vis measurement is the maximal absorption wavelength of vanillin $(280 \mathrm{~nm}), \Delta \mathrm{A}(\Delta \mathrm{A}$ $\left.=\mathrm{A}-\mathrm{A}_{0, \mathrm{~V}}-\mathrm{A}_{0, \mathrm{M}}\right)$ is a negative value. As seen in Fig. 3 , when $\mathrm{n}=1$ or 3 , the plots of $\Delta \mathrm{A} / \mathrm{b}_{0}{ }^{\mathrm{n}}$ versus $\Delta \mathrm{A}$ are curved, while for $\mathrm{n}=2$, the plot of $\Delta \mathrm{A} / \mathrm{b}_{0}{ }^{\mathrm{n}}$ versus $\Delta \mathrm{A}$ is linear, which indicates that 1:2 complexes between vanillin and MAA are formed. In addition, the association constant $(\mathrm{K})$ can be calculated from the slope of the line and the value is $3.12 \times 10^{5} \mathrm{M}^{-2}$, which shows that the formed complexes have considerable stability. After cross-linker and initiator were added to the solution, the complexes were imprinted in the polymeric matrix. Fig. 4 shows the schematic illustration of the molecular imprinting process.

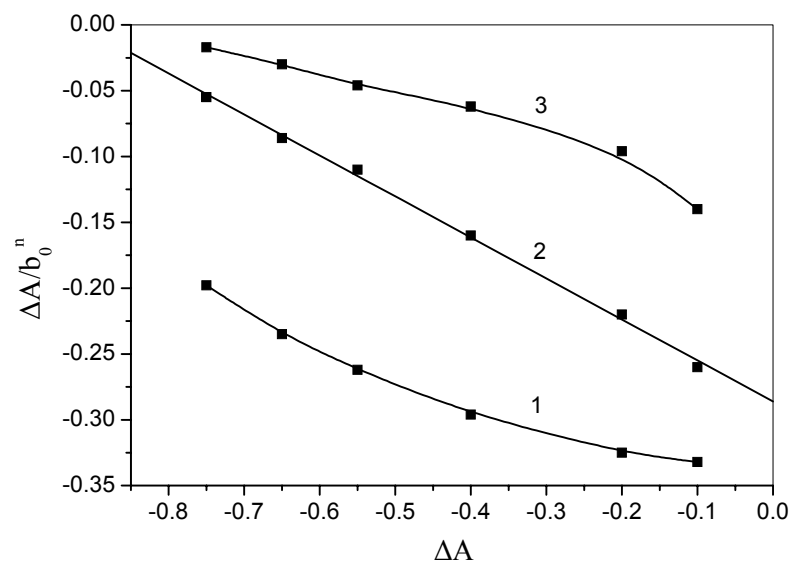

Figure 3: Plot of $\Delta \mathrm{A} / \mathrm{b}_{0}{ }^{\mathrm{n}}$ vs. $\Delta \mathrm{A}$ at $223 \mathrm{~nm}$. Curves: (1) $\mathrm{n}=1$, ordinate: $\Delta \mathrm{A} / \mathrm{b}_{0}\left(\times 10^{3} \mathrm{M}^{-1}\right)$; (2) $n=2$, ordinate: $\Delta \mathrm{A} / \mathrm{b}_{0}{ }^{2}\left(\times 10^{6} \mathrm{M}^{-2}\right) ;(3) \mathrm{n}=3$, ordinate: $\Delta \mathrm{A} / \mathrm{b}_{0}{ }^{3}\left(\times 10^{9} \mathrm{M}^{-3}\right)$. 


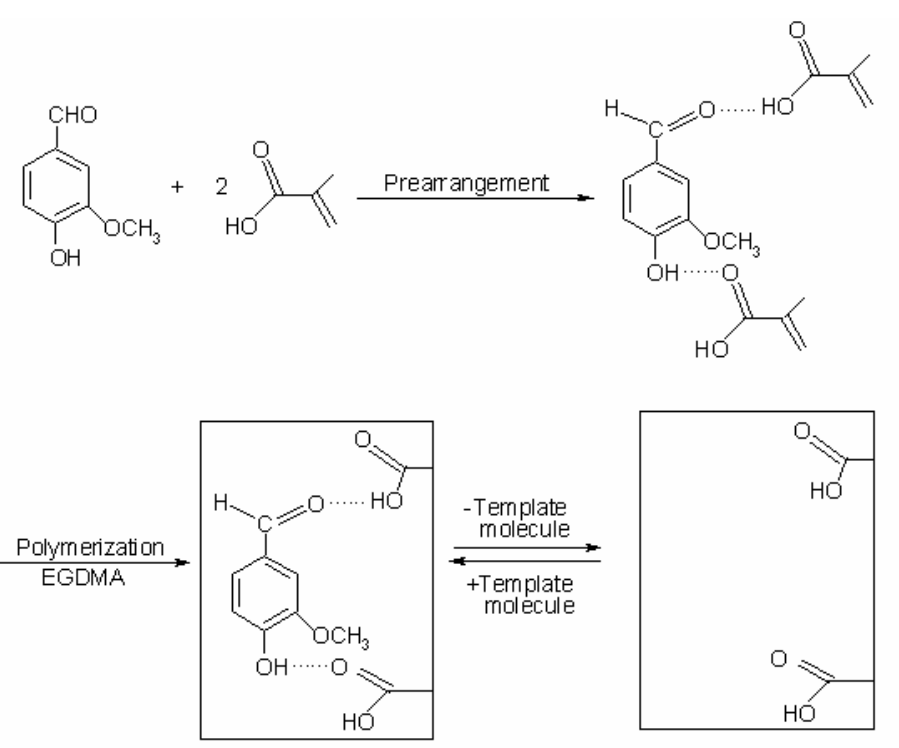

Figure 4: Schematic representation of the molecular imprinting process.

\section{Selective Binding Properties of the Membranes}

In this group of experiments, vanillin and o-vanillin were employed to evaluate the selectivity of the membranes. Fig. 5 presents the molecular structures of vanillin and o-vanillin. As seen in Fig. 5, vanillin and o-vanillin are isomers. In the equilibrium situation, the distribution coefficient $\left(\mathrm{K}_{\mathrm{D}}\right)$ of substrate between membrane and solution rather than the amount of binding substrate onto the membrane was used to characterize the selectivity properties of membranes. Herein, $\mathrm{K}_{\mathrm{D}}$ is expressed as

$$
\mathrm{K}_{\mathrm{D}}=\mathrm{Q}_{\mathrm{m}} / \mathrm{Q}_{\mathrm{s}}
$$

where $\mathrm{Q}_{\mathrm{m}}$ represents the amount of substrate binding to the membrane ( $\mu$ mol.g $\mathrm{g}^{-1}$ ), and $\mathrm{Q}_{\mathrm{s}}$ the equilibrium concentration of substrate in solution $(\mathrm{mM})$. The values of $\mathrm{K}_{\mathrm{D}}$ calculated according to Eq. (10) are shown in Table 1. Compared with the CA-NMIP membrane, the CA-MIP membrane showed high selectivity for substrate. It was noted that $\mathrm{K}_{\mathrm{D}}$ of vanillin on the CAMIP membrane was $31.26 \mathrm{ml.g}^{-1}$, which was almost 3.5 times higher than that on the CA-NMIP membrane and 6.0 times higher than $\mathrm{K}_{\mathrm{D}}$ of o-vanillin on the CA-MIP membrane. Based on the above data analysis, it may be suggested that a comparatively large number of binding sites exist in the CA-MIP membrane, and these binding sites possess the shape of the molecular structure of vanillin. As a result, higher selectivity was exhibited by the CA-MIP membrane and more vanillin was bound to the membrane.

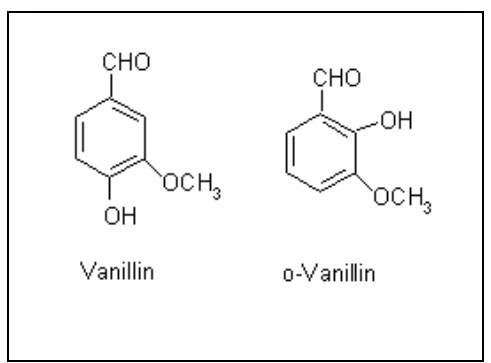

Figure 5: Molecular structures of vanillin and o-vanillin.

Table 1: $K_{D}$ of tested substrates on CA-MIP membrane and CA-NMIP membrane under equilibrium binding conditions

\begin{tabular}{|l|c|c|}
\hline Substrates & K $_{\mathbf{D}}$ on CA-MIP membrane & KD $_{\mathbf{D}}$ on CA-NMIP membrane \\
\hline vanillin & 31.26 & 9.34 \\
o-vanillin & 5.26 & 4.87 \\
\hline
\end{tabular}

Concentration of substrates: $1.00 \mathrm{mM} ; \mathrm{V}=2.0 \mathrm{~mL} ; \mathrm{T}=25^{\circ} \mathrm{C}$; adsorption time: $24 \mathrm{~h}$. 
In order to further understand the binding capacity of vanillin in the CA-MIP membrane, we carried out the binding isotherm and Scatchard analysis for the CA-MIP membrane. Fig. 6 shows the binding isotherm for the CA-MIP membrane; when the concentration of vanillin was varied from 0.1-2.5 mM, the loading amount of vanillin on the CA-MIP membrane increased with the increase of vanillin concentration. It is well known that binding equilibrium constants $\left(\mathrm{K}_{\mathrm{a}}\right)$ rather than binding amount can give more effective information about the substrate binding to the imprinted polymer membrane. Thus, based on the data presented in Fig. 6 , the value of $\mathrm{K}_{\mathrm{a}}$ for the CA-MIP membrane was determined according to the Scatchard equation
(Sihn and Chung, 2003).

$\mathrm{Q} / \mathrm{c}_{\mathrm{e}}=-\mathrm{QK}_{\mathrm{a}}+\mathrm{Q}_{\max } \mathrm{K}_{\mathrm{a}}$

where $c_{e}$ and $Q_{\max }$ represent the free concentration of vanillin at equilibrium and the binding capacity of vanillin to the CA-MIP membrane, respectively. According to Eq. (11), Q/ $\mathrm{c}_{\mathrm{e}}$ was plotted on the vertical axis and $\mathrm{Q}$ on the horizontal axis. The plot of $\mathrm{Q} / \mathrm{c}_{\mathrm{e}}$ versus $Q$ was a straight line (Fig. 7), which indicated that the binding sites in the CA-MIP membrane are equivalent and present equal binding capacity for vanillin. $\mathrm{K}_{\mathrm{a}}$ and $\mathrm{Q}_{\max }$ were determined from the slope and intercept of the line, and the values of $\mathrm{K}_{\mathrm{a}}$ and $\mathrm{Q}_{\max }$ were $2.19 \times 10^{4} \mathrm{M}^{-1}$ and $387.3 \mu \mathrm{mol} / \mathrm{g}$, respectively.

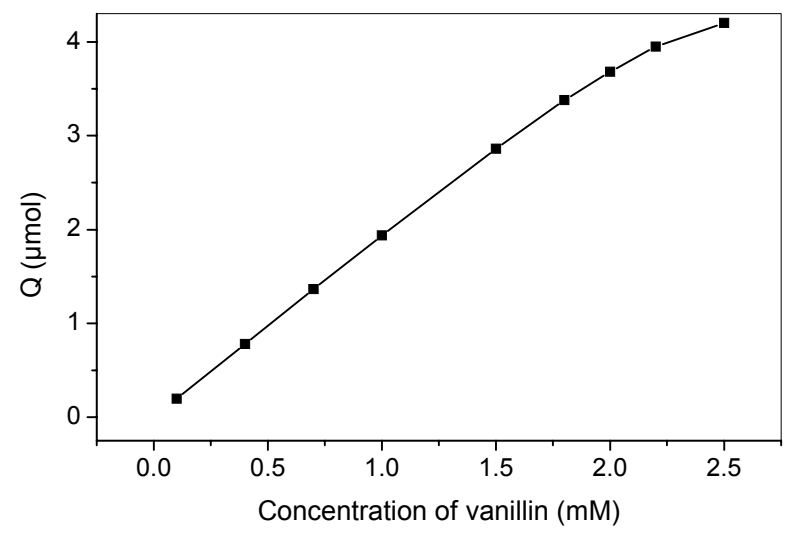

Figure 6: Binding isotherm of vanillin on the CA-MIP membrane. Adsorption time: $24 \mathrm{~h} ; \mathrm{T}=25^{\circ} \mathrm{C} ; \mathrm{V}=2.0 \mathrm{ml}$.

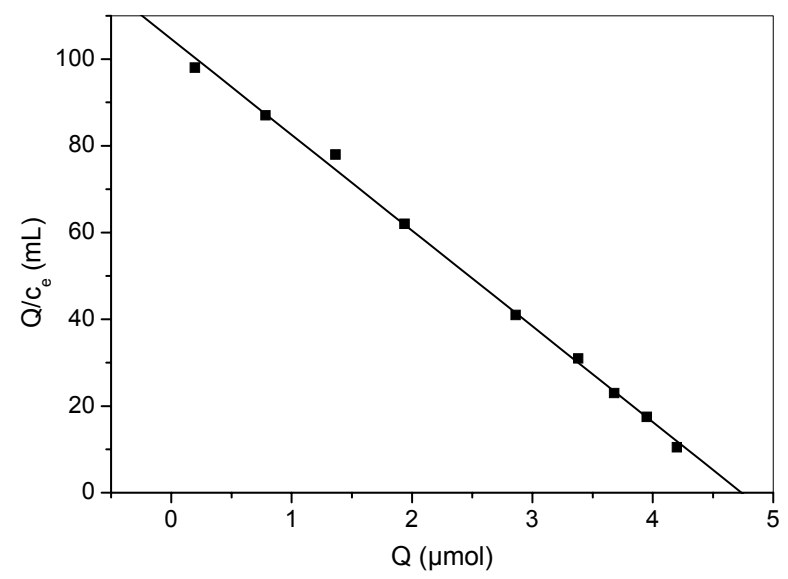

Figure 7: Scatchard plot of $\mathrm{Q} / \mathrm{c}_{\mathrm{e}}$ vs. $\mathrm{Q}$. 


\section{Effect of Cross-Linker Concentration on Vanillin Adsorption on Membranes}

Cross-linker concentration has a direct relationship with vanillin adsorption on membranes. As seen in Fig. 8, compared with the blank CA membrane, the concentration of EGDMA has a significant effect on vanillin binding to CA-MIP and CA-NMIP membranes. An increase of EGDMA concentration up to $320 \mathrm{mM}$ in the reaction mixture lead to an obvious elevation in binding amount of vanillin, while a further increase of EGDMA concentration caused a slight decrease of vanillin binding. It may be suggested that, on the one hand, the imprinted polymer membrane should be rather rigid in order to preserve the structure of the selective cavity after the removal of the template molecule; on the other hand, a certain flexibility of polymer chains should be present to facilitate a fast equilibration with the template molecule in the cavity. Apparently, at increasing levels of vanillin binding, an increase of EGDMA concentration reduces the polymer chain flexibility and thus provides a gain in stabilization of the structure of the selective cavity. This improves the contribution of specific binding to receptor sites and causes an increase of vanillin binding. However, too high of an EGDMA concentration will increase excessively the cross-linker domains, which possess a reduced number of receptor sites. This causes a decrease of vanillin binding for the CA-MIP and CA-NMIP membranes prepared at high EGDMA concentration. Therefore, $320 \mathrm{mM}$ EGDMA was employed to prepare the CA-MIP and CA-NMIP membranes.

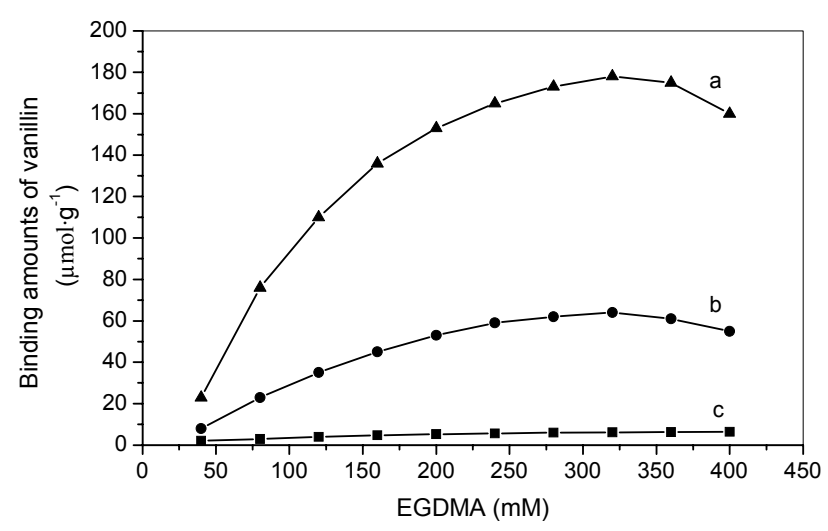

Figure 8: Effect of cross-linker concentration in the reaction mixture on vanillin sorption on (a) CA-MIP membrane; (b) CA-NMIP membrane and (c) blank CA membrane. Binding amounts of vanillin were achieved by immersing membrane in $1.0 \mathrm{mM}$ vanillin solution for $24 \mathrm{~h}$ at $25^{\circ} \mathrm{C}$.

\section{Selective Separation Capacity of Membranes}

In order to better understand separation capacity of polymer membranes, competitive transport experiments with the CA-MIP and CA-NMIP membranes were performed, where the two substrates vanillin and o-vanillin were employed simultaneously. Fig. 9 presents time-transport curves of the two substrates, vanillin and o-vanillin, through CA-MIP membrane and CA-NMIP membrane. Compared with the CA-MIP membrane, diffusion rates of the substrates through the CA-NMIP membrane were slow. As seen from curves a and $\mathrm{c}$ in Fig. 9, the transport rate of vanillin through the CAMIP membrane was obviously higher than that through the CA-NMIP membrane. The distinctly different transport rates of the CA-MIP and CANMIP membranes showed that the molecular imprinting process created channels through which template molecules could pass successfully. In this way, a kind of continuous and fast transport process was accomplished based on selective shape and size and position of functional groups in the channels which could recognize the best suitable template molecule. In contrast, the CA-NMIP membrane did not present any imprinting effect, and the dense and small pores are arranged in a disordered manner that cannot generate channels. In addition, it is also possible that the routeways in the CA-NMIP membrane were jammed, causing vanillin molecules to be transported at a low rate. According to above analysis, it can be suggested that the different 
diffusional behavior of vanillin in the CA-MIP and CA-NMIP membranes results from the molecular imprinting effects. Imprinting creates channels that are not present in the CA-NMIP membrane. Moreover, the effect was also confirmed in curves a and $b$ of Fig. 10, the vanillin molecules were transported faster through the CA-MIP membrane than o-vanillin, showing that the CA-MIP membrane is permselective to the template molecule vanillin. This can be attributed to the molecular imprinting effect of the template molecule vanillin. Thus, after the removal of the template molecule vanillin from the CA-MIP membrane, the shape and size of the pores that exist in the CA-MIP membrane resemble the structure of the vanillin molecule, making the imprinted membrane selective to vanillin and more vanillin were transported through the CA-MIP membrane. In order to further understand the separation capacity of membranes, selectivity factors (ratios of diffusive flux of vanillin to that of o-vanillin) were employed in this group of experiments. As seen in Table 2, the permselectivity of the CA-MIP membrane for vanillin is higher than that of the CA-NMIP membrane by comparing their selectivity factors. The selectivity factor of the CA-MIP membrane for vanillin/o-vanillin is 3.82 , while that of the CA-NMIP membrane is 1.61 after a permeation time of $24 \mathrm{~h}$. This further demonstrates the molecular imprinting effect and shows that the imprinted membranes possess good separation capacity. Therefore, it is suggested that the CA-MIP membranes can be used for the separation and enrichment of vanillin and o-vanillin.

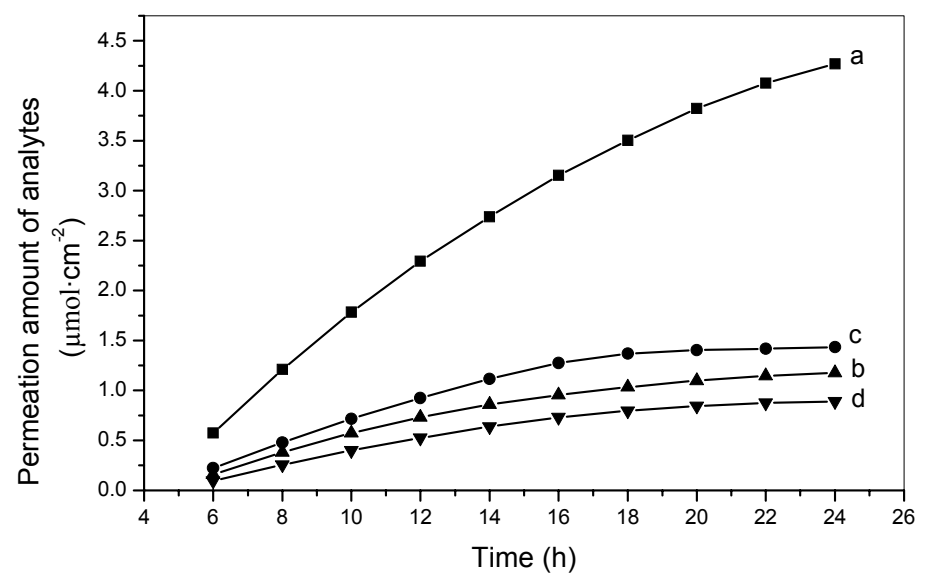

Figure 9: Time-transport curves of the mixture of vanillin and o-vanillin through CA-MIP membrane and CA-NMIP membrane. Curves: (a) vanillin permeate through CA-MIP membrane; (b) o-vanillin permeate through CA-MIP membrane; (c) vanillin permeate through CA-NMIP membrane; (d) o-vanillin permeate through CA-NMIP membrane. Concentration of vanillin and o-vanillin: $1.00 \mathrm{mM}$; V=50 mL; $\mathrm{T}=25^{\circ} \mathrm{C}$; effective area of membranes: $3.14 \mathrm{~cm}^{2}$.

Table 2: Selectivity factors of tested substrates for CA-MIP membranes and CA-NMIP membranes in separate experiments

\begin{tabular}{|c|c|c|}
\hline \multirow{2}{*}{ Membranes } & \multicolumn{2}{|c|}{ Selectivity factors } \\
\hline & vanillin/vanillin & vanillin/o-vanillin \\
\hline CA-MIP membranes & 1.00 & 3.82 \\
\hline CA-NMIP membranes & 1.00 & 1.61 \\
\hline
\end{tabular}

\section{CONCLUSIONS}

Cellulose acetate-based composite microfiltration membranes were prepared successfully using photoinitiated copolymerisation of methacrylic acid (MAA) as functional monomer and ethylene glycol dimethacrylate (EGDMA) as crosslinker in the presence of the template molecule vanillin. The selective binding experiments of substrates indicated that the CA-MIP membranes exhibited high selectivity for vanillin in comparison with o-vanillin, and the maximum binding amount for vanillin was 
$387.3 \mu \mathrm{mol} / \mathrm{g}$. By comparing separation capacity of the CA-MIP and CA-NMIP membranes, it was found that the separation capacity of the CA-MIP membranes was significantly higher than that of the CA-NMIP membranes and a selectivity factor up to 3.82 for vanillin/o-vanillin was achieved. Therefore, it is suggested that such porous imprinted membranes can be very useful for separation of vanillin and o-vanillin.

\section{REFERENCES}

Brauner, N. and Shacham, M., Considering Error Propagation in Stepwise Polynomial Regression, Ind Eng Chem Res, 38, No. 11,4477 (1999).

Chen, C. B., Chen, Y.J. and Zhou, J., A 9vinyladenine-based Molecularly Imprinted Polymeric Membrane for the Efficient Recognition of Plant Hormone ${ }^{1} \mathrm{H}$-indole-3-acetic Acid, Anal Chim Acta, 569, No. 1, 58 (2006).

Cormack, P. A. G. and Mosbach, K., Molecular Imprinting: Recent Developments and the Road Ahead, Reactive Functional Polymers, 41, No.13,115 (1999).

Fan, Z. L., Yang, G. L. and Liu, H.Y., Investigation of Molecularly Imprinted Polymer Rod with Aminoantipyrine as Template by In situ Polymerization Method and Their Separation Characteristics, Chin J Chromatogr, 21, No. 3, 199 (2003).

Haupt, K. and Mosbach, K., Molecularly Imprinted Polymers and Their Use in Biomimetic Sensors, Chem Rev, 100, No. 7, 2495 (2000).

Hilal, N. and Kochkodan, V., Surface Modified Microfiltration Membranes with Molecularly Recognising Properties, J Membr Sci, 213, No. 1, 97 (2003).

Jakusch, M., Janotta, M. and Mizaikoff, B., Molecularly Imprinted Polymers and Infrared Evanescent Wave Spectroscopy, A Chemical Sensors Approach, Anal Chem, 71, No. 20,4786 (1999).

Jodlbauer, J., Maier, N.M. and Lindner, W., Towards Ochratoxin A Selective Molecularly Imprinted Polymers for Solid-phase Extraction, J Chrogatogr A. 945, No. 1, 45 (2002).

Kochkodan, V., Weigel, W. and Ulbricht, M., Thin Layer Molecularly Imprinted Microfiltration Membranes by Photofunctionalization Using a Coated $\alpha$-Cleavage Photoinitiator, Analyst, 126, No. 6, 803 (2001).
Kroger, S., Turner, A. P. F. and Mosbach, K., Imprinted Polymer-based Sensor System for Herbicides Using Differential-pulse Voltammetry on Screen-printed Electrodes, Anal Chem, 71, No. 17, 3698 (1999).

Kugimiya, A. and Takeuchi, T., Application of Indoleacetic Acid-imprinted Polymer to Solid Phase Extraction, Anal Chim Acta, 395, No. 3, 251 (1999).

Mullett, W. M. and Lai, E. P. C., Determination of Theophylline in Serum by Molecularly Imprinted Solid-phase Extraction with Pulsed Elution, Anal Chem, 70, No. 17, 3636 (1998).

Piletsky, S. A., Matuschewski, H. and Schedler, U., Surface Functionalization of Porous Polypropylene Membranes with Molecularly Imprinted Polymers by Photograft Copolymerization in Water, Macromolecules, 33, No. 8, 3092 (2000).

Sellergren, B., Wieschemeyer, J. and Boos, K. S., Imprinted Polymers for Selective Adsorption of Cholesterol from Gastrointestinal Fluids, Chem Mater, 10, No.12, 4037 (1998).

Sihn, Y. S. and Chung H. S., Interpretations of the TDxFLx $^{\circledR}$ Calibration Data of the Abused Drugs, Forensic Science International, 131, No. 1, 1 (2003).

Takeda, K. and Kobayashi, T., Hybrid Molecularly Imprinted Membranes for Targeted Bisphenol Derivatives, J Membr Sci, 275, No. 1-2, 61 (2006).

Trotta, F., Drioli, E. and Baggiani, C., The Use of Trace Ions for Advanced Characterisation of Transport Properties of NF Membranes in Electrolyte Solutions: Theoretical Analysis, J Membr Sci, 201, No. 1, 77 (2002).

Vlatakis, G., Andersson, L.I. and Muller, R., Drug Assay Using Antibody Mimics Made by Molecular Imprinting, Nature, 361, No.6413, 645 (1993).

Xu, X. J., Zhu, L. L. and Chen, L.R., Separation and Screening of Compounds of Biological Origin Using Molecularly Imprinted Polymers, J Chromatogr B, 804, No.1,61 (2004).

Yoshikawa, M., Murakoshi, K. and Kogita, T., Chiral Separation Membranes from Modified Polysulfone Having Myrtenal-derived Terpenoid Side Groups, European Polymer Journal, 42, No. 10, 2532 (2006).

Zhou, J., He, X. W. and Yang, W. L, Studies on Character of Adsorption and Recognition of Etharidine Template Polymer, Chem. J Chin Univ., 19, No. 9, 1388 (1998). 